\title{
Suprachiasmatic Nuclei Grafts Restore the Circadian Rhythm in the Paraventricular Nucleus of the Hypothalamus
}

\author{
Ehab Tousson and Hilmar Meissl \\ Max Planck Institute for Brain Research, 60528 Frankfurt am Main, Germany
}

The mammalian suprachiasmatic nucleus (SCN) controls the circadian rhythm of many physiological and behavioral events by an orchestrated output of the electrical activity of SCN neurons. We examined the propagation of output signals from the SCN into the hypothalamus, especially into the region of the paraventricular nucleus, through multimicroelectrode recordings using acute and organotypic brain slices. Circadian rhythms in spontaneous firing rate with a period close to $24 \mathrm{hr}$ were demonstrated in the SCN, in directly adjacent hypothalamic regions, and in the region of the paraventricular nucleus of the hypothalamus, an important center for the integration of neuroendocrine, homeostatic, and autonomic functions. The activity rhythms recorded from structures outside of the SCN were in phase with the rhythms in the SCN. Cyclic information in the hypothalamus was lost after ablation of the SCN but could be restored by SCN grafts, indicating that a humoral factor is responsible for the restoration of circadian rhythmicity in the absence of neural connections. Periodic application of arginine-vasopressin (AVP) provided evidence that AVP can induce rhythmicity in the hypothalamus. These data indicate that the SCN uses a dual (neuronal and humoral) mechanism for communication with its targets in the brain.

Key words: suprachiasmatic nucleus; paraventricular nucleus; hypothalamus; circadian rhythms; multimicroelectrode recordings; vasopressin

\section{Introduction}

Over the past few years, a compelling picture of the suprachiasmatic nucleus (SCN) clockwork has been established (Reppert and Weaver, 2001). The molecular clock mechanism involves interacting positive and negative transcriptional feedback loops that drive recurrent rhythms in the RNA and protein levels of key clock components (Reppert and Weaver, 2002). Photic cues reflecting the environmental light/dark cycle are the most important entraining signals connecting the clockwork with the external time. Recent studies have provided evidence for the involvement of classical (Aggelopoulos and Meissl, 2000; Lucas et al., 2003) and nonrod, noncone retinal photoreceptors (Freedman et al., 1999; Berson et al., 2002) in circadian photoreception. Although the progress in elucidating the molecular clockwork mechanism and its regulation have been spectacular, the knowledge of how these molecular loops are interpreted by the numerous clock cells and how they lead to concerted output signals that can synchronize endocrine and autonomic centers in the hypothalamus is very limited.

Because of the widespread and prominent neural projections of the SCN to numerous neuroendocrine and autonomic structures in the brain, its neural output is regarded as the primary

Received Nov. 13, 2003; revised Dec. 23, 2003; accepted Jan. 22, 2004.

We thank Heinz Waessle for continuous support and discussion of this study. We also thank F. Boij for expert technical assistance, Arlene Hirano (Department of Neurobiology, University of California Los Angeles, Los Angeles, CA) for critically reading this manuscript, and Paul Pevet (Department of Neurosciences, Strasbourg, France) and Peter Ekstrom (Institute of Cell and Organism Biology, Lund, Sweden) for helpful comments.

Correspondence should be addressed to Hilmar Meissl, Department of Neuroanatomy, Max Planck Institute for Brain Research, Deutschordenstrasse 46, 60528 Frankfurt am Main, Germany. E-mail: meiss@@mpih-frankfurt.mpg.de.

DOl:10.1523/JNEUROSC1.5044-03.2004

Copyright $\odot 2004$ Society for Neuroscience $\quad 0270-6474 / 04 / 242983-06 \$ 15.00 / 0$ mechanism for transmitting cyclic information to the brain (Reppert and Weaver, 2002). However, transplantation of SCN grafts into SCN-lesioned animals can restore circadian locomotor activity rhythmicity (Ralph et al., 1990), possibly by a diffusible signal from the SCN graft (Silver et al., 1996). Although circadian locomotor rhythms are restored by SCN transplants, it appears that circadian neuroendocrine rhythms are not reestablished by the grafts (Meyer-Bernstein et al., 1999). Rather, they appear to require intact neural projections from the SCN that might be either inhibitory or stimulatory (Kalsbeek et al., 2000; Perreau-Lenz et al., 2003).

The present study was initiated to dissociate neuronal and humoral circadian output signals from the SCN that are used for communication with the targets in the brain.

\section{Materials and Methods}

Animals and experimental procedure. Care of animals and all experiments were conducted in accordance with institutional guidelines and with local ethical approval.

In the present study, we used planar multimicroelectrode arrays (MEAs) to perform long-term recordings of the extracellular electrical activity from acute $(n=21)$ or organotypic $(n=18)$ brain slices of the mouse. In brief, hypothalamic tissue was prepared from CD1 mice (5-6 weeks old for acute slices; $2-4 \mathrm{~d}$ old for organotypic slices). The mice were bred in the animal facility of the Max Planck Institute under controlled conditions (12 hr light/dark cycle; lights on at 6:00 A.M.). The brain was removed after decapitation and placed in ice-cold artificial CSF (124 mm NaCl, $5 \mathrm{~mm} \mathrm{KCl,} 1.25 \mathrm{~mm} \mathrm{KH} \mathrm{PO}_{4}, 1.3 \mathrm{~mm} \mathrm{MgSO}$, $26 \mathrm{~mm}$ $\mathrm{NaHCO}_{3}, 2.2 \mathrm{~mm} \mathrm{CaCl}_{2}, 10 \mathrm{~mm}$ glucose, $10 \mathrm{~mm}$ HEPES, $100 \mathrm{U} / \mathrm{ml}$ penicillin, $100 \mu \mathrm{g} / \mathrm{ml}$ streptomycin, and $1 \mu \mathrm{g} / \mathrm{ml}$ amphotericin B). Coronal slices of $300 \mu \mathrm{m}$ thickness were made using a vibratome. Explants limited to the relevant hypothalamic areas were dissected with a pair of scalpels 
using the optic chiasm and the third ventricle as landmarks. Selected sections were trimmed dorsally at the level of the paraventricular thalamic nucleus and laterally just before the supraoptic nuclei. The selected slice was then positioned over the poly-D-lysine-coated MEA and incubated at $37^{\circ} \mathrm{C}$ in $5 \% \mathrm{CO}_{2}-95 \%$ air with a small amount of culture medium for $1 \mathrm{hr}$ before the electrode array was filled with medium. In acute slice experiments, recordings started 3-5 hr after dissection; in organotypic slice experiments, the tissue was incubated for $>3$ weeks before recording.

Culture medium, which consisted of DMEM-Ham's F12 (Invitrogen, San Diego, CA) supplemented with 10\% fetal calf serum, 10 mM HEPES, $100 \mathrm{U} / \mathrm{ml}$ penicillin, $100 \mu \mathrm{g} / \mathrm{ml}$ streptomycin, and $100 \mu \mathrm{g} / \mathrm{ml}$ ascorbic acid, was exchanged every $2-3 \mathrm{~d}$. The recording medium was similar to the culture medium with the exception of the HEPES content, which was increased to $20 \mathrm{~mm}$, the $\mathrm{NaHCO}_{3}$ level was reduced to $0.56 \mathrm{gm} / \mathrm{l}$, and 4 $\mu \mathrm{l} / \mathrm{ml}$ amphotericin B was added. During the recordings, medium was exchanged continuously at a flow rate of $5 \mathrm{ml}$ per hour using a modified perfusion pump (Braun, Melsungen, Germany). Recordings of spontaneous discharges from different parts of the hypothalamus were performed at $36^{\circ} \mathrm{C}$ with a MEA-1060 recording system using MC_Rack software (Multi Channel Systems, Reutlingen, Germany). Multimicroelectrode arrays consisted of 60 electrodes of $30 \mu \mathrm{m}$ diameter arranged in an $8 \times 8$ pattern. The signals from the MEA electrodes were amplified $\times 1200$ and sampled at $25 \mathrm{kHz}$ per channel on 60 channels simultaneously. Spikes were counted using a spike detector and sampled in $6 \mathrm{~min}$ intervals for up to 2 weeks. In acute and organotypic slice preparations of the hypothalamus, we usually observed multiunit spike signals with a signal-to-noise ratio that often did not allow the discrimination between single units. The amplitude of the rhythm was usually larger in acute slice preparations compared with organotypic slices, but the latter have the advantage that they permit longer recording periods. To ascertain that we were reliably recording spike potentials, we routinely blocked spike activity by bath application of $200 \mathrm{~nm}$ tetrodotoxin (TTX) during the experiments. Because sodium-dependent action potentials represent an output signal of the endogenous oscillator but are not part of the timekeeping mechanism, the block of these potentials does not interrupt the rhythm (Shibata and Moore, 1993; Welsh et al., 1995). The orientation of the brain slice on the multimicroelectrode array was documented at the beginning of the experiments with a digital camera. After electrophysiological recordings, the brain slices were fixed in $4 \%$ paraformaldehyde in $0.1 \mathrm{~m}$ phosphate buffer, $\mathrm{pH} 7.4$, and cut on a cryostat $(20 \mu \mathrm{m})$; sections were then stained with cresyl violet or immunostained for neurochemicals characteristic of the SCN or the paraventricular nucleus of the hypothalamus (PVN) [GABA and vasoactive intestinal polypeptide; Sigma, St. Louis, MO; arginine-vasopressin (AVP), kindly provided by F. F. Nuernberger, Department of Anatomy, Frankfurt am Main] to verify the electrode locations and also to verify the completeness of $\mathrm{SCN}$ ablation in the graft experiments.

Analysis of circadian rhythms and statistics. Circadian periods and statistics were determined using Cosinor analyses (Time Series AnalysisSeriel Cosinor; Expert Soft Technologie, Esvres, France). In the histograms (see Figs. 1-4), data were smoothed by a moving average algorithm (IDL software; Research Systems, Boulder, CO).

\section{Results}

\section{Propagation of circadian signals in the hypothalamus}

We describe here circadian neural rhythms in coronal hypothalamic brain slices prepared from CD1 mice. For this purpose, we have developed a method to culture acute and organotypic brain slices for long periods of up to 2 weeks on planar multimicroelectrode arrays; only experiments with successful recordings lasting $>5 \mathrm{~d}$ were included in this study. In this way it was possible to probe many neurons simultaneously in a relatively intact hypothalamic network for long periods. Application of drugs in control experiments that block spike propagation (e.g., tetrodotoxin) or drugs that phase shift the circadian clock (e.g., melatonin, pituitary adenylate cyclase-activating polypeptide) indicated that the substrate-embedded microelectrode arrays reliably recorded
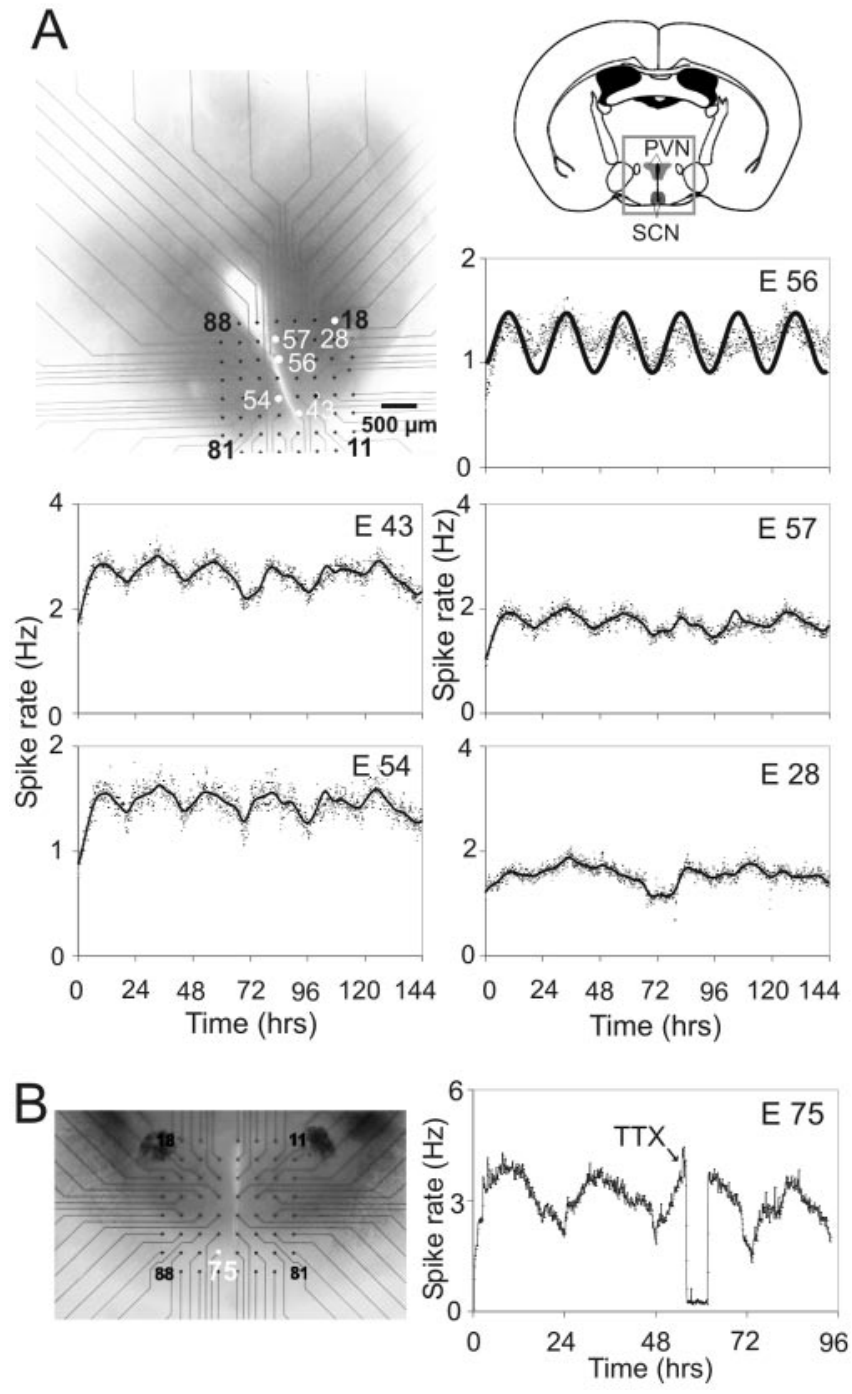

Figure 1. Representative circadian rhythms of multiunit activity recorded from the hypothalamus of an organotypic slice preparation. The diagram in the top left corner shows the approximate extension of the slice with the $S C N$ and the PVN in a frame. $A$, Recordings from the $\mathrm{SCN}(\mathrm{E} 43, \mathrm{E} 54)$, the region of the PVN (E56, E57), and the lateral hypothalamus (E28). The dots in the histograms represent the average multiunit activity sampled in 6 min intervals (spike rate). The continuous line in E56 depicts a fit with a cosine function; in the other recordings, data were smoothed (continuous line; see Materials and Methods). B, Control experiment with $1 \mathrm{hr}$ application of $200 \mathrm{~nm}$ tetrodotoxin (arrow).

neural spike activity (data not shown). Clear circadian rhythms in spontaneous firing rate with periods near $24 \mathrm{hr}$ (acute slices, $24.03 \pm 0.27, n=21$; organotypic slices, $23.75 \pm 0.23, n=18$ ) were observed not only in the region of the SCN and in directly adjacent hypothalamic areas but also in the dorsal hypothalamus, in the subparaventricular zone, in the PVN, and in regions in which descending paraventricular pathways have been described. Recordings from acute and organotypic brain slices from the SCN and PVN gave essentially similar results concerning the distribution of circadian signals and the phase of the rhythms.

The present study focuses on the SCN and the zone of the PVN; therefore, Figure 1 shows some representative examples of recordings only from these hypothalamic areas of an organotypic brain slice (suprachiasmatic area, electrodes E43 and E54; the region of the PVN, electrodes E56 and E57; as a control, a nonrhythmic area in the lateral hypothalamus, E28). The histogram 
in E56 shows a cosine curve with a $24 \mathrm{hr}$ period calculated by Cosinor analysis; in the other histograms, data were smoothed by a moving average algorithm. In all experiments, Cosinor analysis was used to calculate circadian rhythm parameters, including mesor, amplitude, and acrophase. It appears from these recordings that the subparaventricular zone and the area of the PVN receive direct or indirect projections from the $\mathrm{SCN}$, as suggested previously using immunocytochemistry combined with tracing techniques (Vrang et al., 1995). The spatial resolution of the multielectrode system did not allow discrimination between the different subnuclei in the PVN. Interestingly, in all of our experiments, the activity rhythms recorded from structures outside of the SCN are in phase with the rhythms in the SCN. We never observed in an in vitro preparation a clear phase difference or a reversal of multiunit activity between the SCN and the area of the paraventricular nucleus of the hypothalamus or the subparaventricular zone, as described in vivo in previous reports (Inouye and Kawamura, 1979; Kubota et al., 1981).

\section{SCN grafts can restore rhythmicity in the paraventricular nucleus of the hypothalamus}

In the next series of experiments, we surgically isolated the region of the PVN with unilateral or bilateral knife-cuts that removed one SCN or both nuclei from acute brain slices and positioned the remaining hypothalamus with the region of the PVN on the MEA. In brain slices with a unilateral removal of the SCN (data not shown), circadian rhythmicity was still observed in the PVN and other hypothalamic regions, but bilateral ablation of the SCN completely abolished all rhythms in the PVN and in adjacent hypothalamic areas (Fig. $2 A, B$ ). These results suggest that the circadian rhythmicity in the hypothalamus depends on the presence of an intact SCN. The question of why the circadian rhythmicity was not completely abolished on one side of the hypothalamus when the SCN was unilaterally removed was addressed in another series of experiments. Hypothalamic slices with an ablation of the SCN were placed on multimicroelectrode arrays in the same way as in previous experiments. The completeness of the SCN ablation was verified after the experiments by histological and immunocytochemical investigations of the sections. SCN tissue was cultured separately on filter membranes. Figure 2 depicts one of these experiments, with the hypothalamic PVN on the multielectrode array and an SCN graft on a filter membrane (right), showing clearly the nuclei and the optic chiasm. The SCN tissue, prepared in the morning of the day of the graft experiment, was added at two different times to the recording chambers: directly in the beginning (Fig. $2 \mathrm{~A}$ ) and after 2 or $3 \mathrm{~d}$ of recordings from the PVN (Fig. $2 B$ ). When we started cocultures of PVN and SCN from the beginning of the experiment, there was a clear circadian rhythmicity in the PVN and adjacent areas. The rhythmicity disappeared when the SCN tissue was removed from the array (Fig. $2 \mathrm{~A}$ ). In hypothalamic slices that received an SCN graft 2 or $3 \mathrm{~d}$ after the onset of the experiments, the previously irregular firing rate of PVN neurons, which showed no signs of a $24 \mathrm{hr}$ rhythm, was synchronized and exhibited a clear circadian rhythmicity as long as the cocultures with SCN tissue persisted (Fig. $2 B$ ). Peak activity was attained in both experiments $>7 \mathrm{hr}$ after addition of the graft. The observation that SCN transplants can reinstate a neuronal rhythm in the PVN and other hypothalamic targets of the SCN raises questions regarding the nature of the signals by which the SCN expresses its clock function. In the present experiment, we can exclude that the SCN grafts established neural synaptic connections with the hypothalamic slice on the multielectrode arrays. Brain slices and grafts were
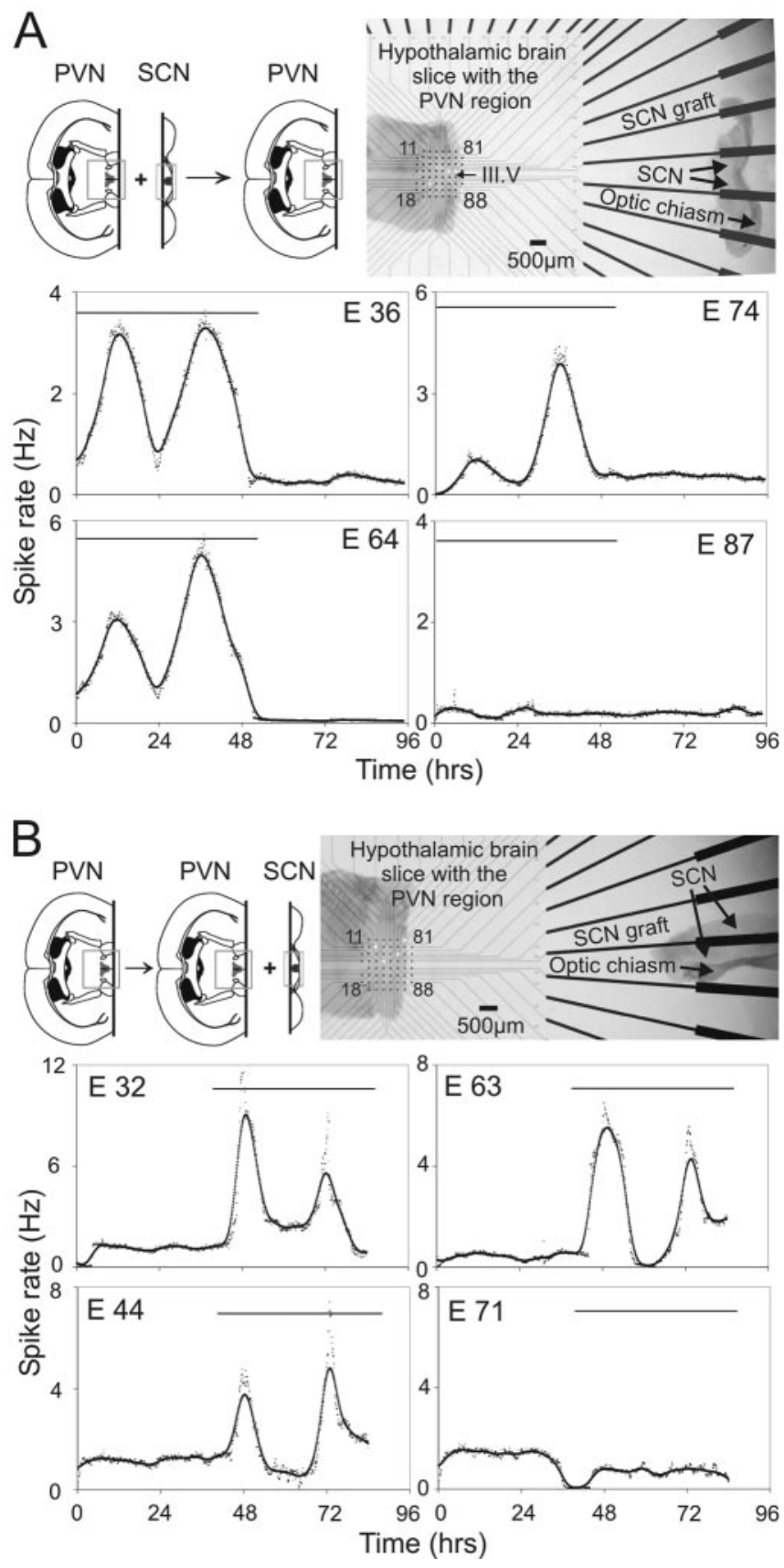

Figure 2. Recordings from acute hypothalamic brain slices with the region of the PVN positioned on the MEA but with SCN ablation. The SCN grafts, cultured separately on pieces of sterile Millicell-CM membranes (Millipore, Bedford, MA), were added at two different times to the recording chamber: directly at the beginning of an experiment $(A)$ and after $44 \mathrm{hr}(B)$. The experimental procedure is shown in the small schematics. The black bar indicates the period of cocultures of PVN and SCN. The clear circadian rhythmicity $(A)$ in the region of the PVN cocultured with $\mathrm{SCN}$ grafts $(n=7)$ disappears after removal of the $S \mathrm{CN}$ graft. When an $\mathrm{SCN}$ graft is added after $44 \mathrm{hr}$ to the recording chamber $(B)$, a $24 \mathrm{hr}$ rhythm is induced in neurons of the isolated PVN $(n=4)$. Both experiments show representative recordings from three electrodes in the region of the PVN with rhythmicity and one electrode in the lateral hypothalamus without induction of rhythm.

mounted on different substrates. The free-floating filter membrane with the SCN graft was usually located at the edge of the culture chamber, whereas the brain slice was centered on the MEAs with a distance of $>10 \mathrm{~mm}$ between both tissues. Induction of a circadian rhythm or activity loss started within a few hours after addition or removal of the graft. Visual inspection of 


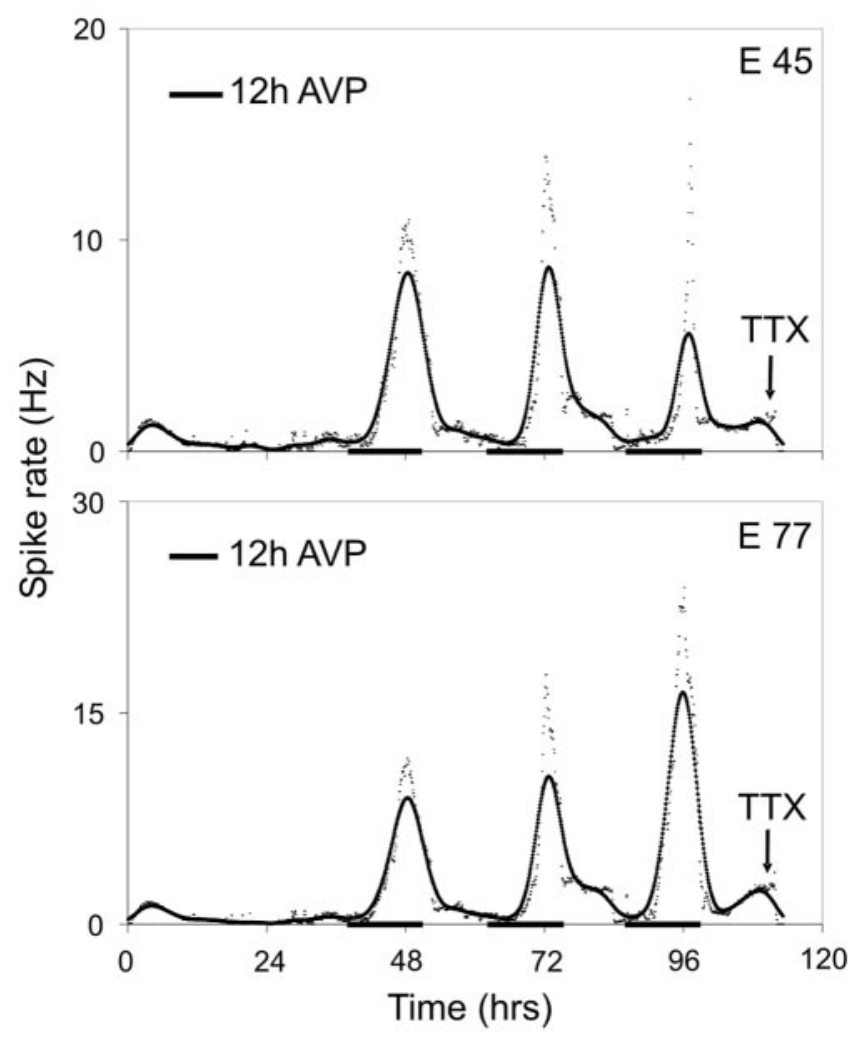

Figure 3. Recordings from two representative electrodes in the PVN region of the hypothalamus after ablation of the SCN. Repeated application of $200 \mathrm{~nm}$ AVP in $12 \mathrm{hr}$ intervals (black bars) followed by $12 \mathrm{hr}$ AVP-free medium induced a rhythm of $\sim 24 \mathrm{hr}(n=4)$. In some electrodes, there is a small increase in activity when the superfusion starts but no peak $24 \mathrm{hr}$ later.

the slices under an inverted microscope never revealed connecting fibers between the explants or neural outgrowth from the graft. Cresyl violet staining and immunocytochemical analysis of the brain slice and the graft at the end of the experiments helped to ascertain the electrode locations, the completeness of SCN ablation, as well as the integrity of the SCN graft.

\section{Arginine-vasopressin can mimic the effect of an SCN graft}

The previous experiments indicated that a diffusible signal could be responsible for the induction of circadian rhythmicity in the hypothalamic PVN when it was cocultured with SCN grafts. There is evidence that AVP in the rodent SCN is involved in regulating timing programs of locomotor behavior (MeyerBernstein et al., 1999; Jansen et al., 2000). AVP release from the SCN shows a robust circadian rhythm in vivo and in vitro (Earnest and Sladek, 1986; Kalsbeek et al., 1995), and the secretion of vasopressin seems to follow the general pattern of electrical activity of SCN neurons (Gillette and Reppert, 1987). We therefore investigated in acute brain slices whether AVP could replace the diffusible signal released from the graft (Fig. 3). Hypothalamic slices containing the PVN were cultured after ablation of the SCN on multimicroelectrode plates. AVP was added in a concentration $(200 \mathrm{~nm})$ that was shown previously to exert short-term effects in the PVN (Inenaga and Yamashita, 1986; Hermes et al., 2000 ) in periodic intervals ( $12 \mathrm{hr}$ application followed by a $12 \mathrm{hr}$ pause) to the superfusion medium. Figure 3 shows two representative recordings from the PVN, where application of AVP could induce, with a considerable time lag (peak activity was attained after $>10 \mathrm{hr}$ ), rhythmicity in the PVN that clearly followed the
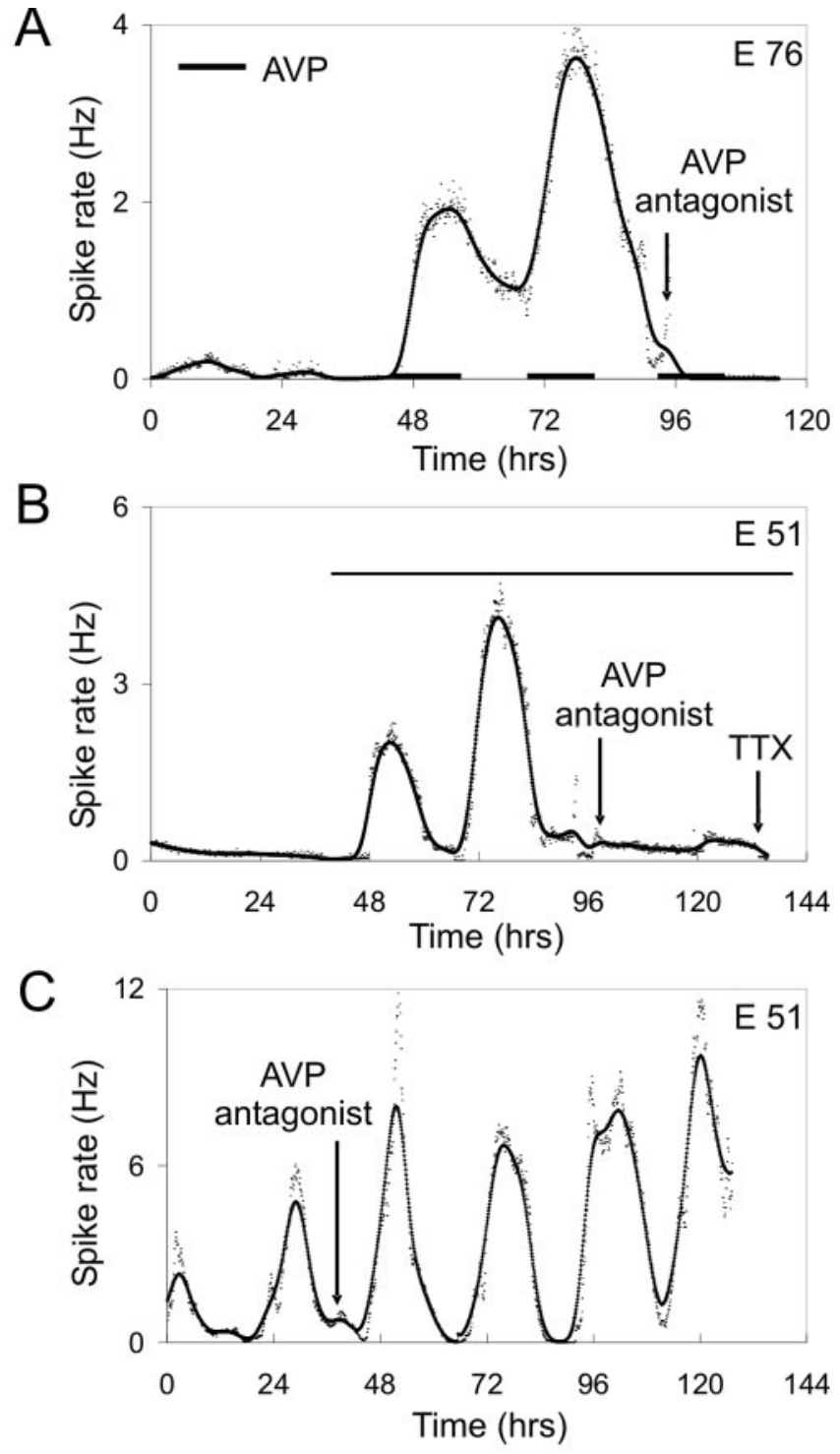

Figure 4. Recordings of multiunit activity from the PVN of the hypothalamus. A, Periodic application of AVP ( $200 \mathrm{~nm})$ induces rhythmicity in the PVN. The rhythm disappears when an AVP receptor antagonist (arrow) is simultaneously applied $(n=3)$. $B$, The figure shows that a rhythm in the isolated PVN that was restored by an SCN graft is completely blocked by $24 \mathrm{hr}$ application of a $1 \mu \mathrm{m}$ concentration of an AVP antagonist (arrows; $n=4$ ). $C$, In an intact brain slice, application of an AVP receptor antagonist (1 $\mu \mathrm{M}$ ) for $24 \mathrm{hr}$ (arrow) has almost no effect on the circadian rhythm recorded from the PVN $(n=6)$. In this experiment, the $S C N$ was located outside of the electrode array but had an intact neural connection with the PVN.

application pattern of AVP; the spike rate then declined in the presence of AVP. The induction of rhythmicity in the PVN by AVP was completely blocked by simultaneous application of a $1 \mu \mathrm{M}$ concentration of the $\mathrm{V}_{1}$ and $\mathrm{V}_{2}$ vasopressin receptor antagonist (1-mercaptocyclohexyl)acetyl-Tyr $(O$-Ethyl)-PheVal-Asn-Cys-Pro-Arg- $\mathrm{NH}_{2}$ (Sigma) (Fig. 4A). Furthermore, the restoration of a circadian rhythm in the PVN cocultured with an SCN graft was also abolished by the AVP receptor antagonist (Fig. $4 B$ ). Surprisingly, in recordings from the PVN in intact brain slices, circadian rhythms remained undisturbed by a $24 \mathrm{hr}$ treatment with the AVP receptor antagonist (Fig. $4 C$ ), showing that under normal conditions, the neural signal seems to over-ride the humoral signal. 


\section{Discussion}

The present results demonstrate that the rhythmic activity in the area of the PVN of the hypothalamus, a prime center for the control of both the neuroendocrine and autonomic systems, is controlled by inputs from the SCN. This input may be either neural, by efferent projections from the SCN, or humoral, by a diffusible factor released from the SCN into the CSF or extracellular space. In slice preparations of the hypothalamus with intact neural connections, the neural efferent output from the SCN seems to determine the output signal, but under experimental conditions in which the efferent connections are interrupted, a diffusible factor can clearly induce rhythmicity in the hypothalamus.

We provide the first evidence that AVP might be a candidate for this diffusible agent. AVP can mimic the action of the humoral factor on the PVN. This action could be blocked by a vasopressin receptor antagonist, showing that the process is receptor mediated. Vasopressin receptors have been identified previously in the hypothalamic PVN and in the SCN (DuboisDauphin et al., 1996; Hurbin et al., 1998). It has been suggested that AVP acts as a feedback regulator of electrical activity in the SCN and has a role in amplifying rhythmicity in the nucleus, but AVP also functions as an important output signal of the SCN (Ingram et al., 1998). The SCN contains numerous AVPimmunoreactive neurons in the dorsomedial subdivision of the nucleus that project to other hypothalamic areas, including the ventral PVN and the dorsal parvicellular subdivision of the PVN (Abrahamson and Moore, 2001). SCN lesions result in the disappearance of AVP-containing fibers in the region of the PVN (Hoorneman and Buijs, 1982). Additionally, there is evidence that vasopressin is released in a circadian pattern from the SCN as a humoral signal. This circadian pattern is lost and vasopressin levels in the CSF are strongly reduced in SCN-lesioned animals, whereas neither lesion of the PVN nor knife-cuts interrupting most neural efferents from the SCN had this effect (Schwartz and Reppert, 1985). In the present study, we cannot exclude the possibility that the effect of the diffusible agent reflects a passive process caused by a leakage from degenerating neurons of the graft. AVP is believed to act through an increase in GABAergic inhibition on PVN neurons (Hermes et al., 2000), whereas Inenaga and Yamashita (1986) have described excitatory AVP responses in the PVN. Gouzenes et al. (1998) demonstrated that AVP exerted inhibitory or excitatory effects according to their initial pattern of discharge. However, the long latency of the induction of rhythmicity argues against a simple role of AVP as efferent neurotransmitter. Previous studies have shown that AVP may have a functional role in the control of the sleep-wake rhythm and the diurnal rhythms of corticosterone secretion. Infusion of AVP or AVP antagonists with an osmotic pump into the third ventricle significantly altered the sleep-wake pattern in the rat (Kruisbrink et al., 1987). It was also shown that the AVP levels in the CSF affect the arousal state of the rat in the dark period (Arnauld et al., 1989). Microinfusions of AVP into the PVN region showed a strong inhibitory effect on corticosterone release of SCN-lesioned animals, indicating a role for AVP in the hypothalamo-pituitary-adrenal axis, possibly by influencing corticotropin-releasing hormone secretion of PVN neurons (Kalsbeek et al., 1992). Transneuronal tracings combined with physiological experiments have also provided evidence for a second polysynaptic pathway with a central role for the hypothalamic PVN, involving the retina, SCN, hypothalamus, sympathetic nervous system, and adrenal cortex. It was concluded that the SCN uses a dual mechanism for corticosterone secretion (and possibly also for other endocrine functions): a direct control of hypothalamic neuroendocrine neurons as well as of neurons of the autonomic nervous system (Buijs and Kalsbeek, 2001).

The present study provides evidence for an additional pathway, a humoral factor that couples the circadian output signal of the SCN to its target areas in the hypothalamus.

\section{References}

Abrahamson EE, Moore RY (2001) Suprachiasmatic nucleus in the mouse: retinal innervation, intrinsic organization and efferent projections. Brain Res 916:172-191.

Aggelopoulos NC, Meissl H (2000) Responses of neurones of the rat suprachiasmatic nucleus to retinal illumination under photopic and scotopic conditions. J Physiol (Lond) 523:211-222.

Arnauld E, Bibene V, Meynard J, Rodriguez F, Vincent JD (1989) Effects of chronic icv infusion of vasopressin on sleep-waking cycle of rats. Am J Physiol 256:R674-R684.

Berson DM, Dunn FA, Takao M (2002) Phototransduction by retinal ganglion cells that set the circadian clock. Science 295:1070-1073.

Buijs RM, Kalsbeek A (2001) Hypothalamic integration of central and peripheral clocks. Nat Rev Neurosci 2:521-526.

Dubois-Dauphin M, Barberis C, De Bilbao F (1996) Vasopressin receptors in the mouse (Mus musculus) brain: sex-related expression in the medial preoptic area and hypothalamus. Brain Res 743:32-39.

Earnest DJ, Sladek CD (1986) Circadian rhythms of vasopressin release from individual rat suprachiasmatic explants in vitro. Brain Res 382:129-133.

Freedman MS, Lucas RJ, Soni B, von Schantz M, Munoz M, David-Gray Z, Foster R (1999) Regulation of mammalian circadian behavior by nonrod, non-cone, ocular photoreceptors. Science 284:502-504.

Gillette MU, Reppert SM (1987) The hypothalamic suprachiasmatic nuclei: circadian patterns of vasopressin secretion and neuronal activity in vitro. Brain Res Bull 19:135-139.

Gouzenes L, Desarmenien MG, Hussy N, Richard P, Moos FC (1998) Vasopressin regularizes the phasic firing pattern of rat hypothalamic magnocellular vasopressin neurons. J Neurosci 18:1879-1885.

Hermes MLHJ, Ruijter JM, Klop A, Buijs RM, Renaud LP (2000) Vasopressin increases GABAergic inhibition of rat hypothalamic paraventricular nucleus neurons in vitro. J Neurophysiol 83:705-711.

Hoorneman EMD, Buijs RM (1982) Vasopressin fiber pathways in the rat brain following suprachiasmatic nucleus lesioning. Brain Res 243:235-241.

Hurbin A, Boissin-Agasse L, Orcel H, Rabie A, Joux N, Desarmenien MG, Richard P, Moos FC (1998) The $\mathrm{V}_{1 \mathrm{a}}$ and $\mathrm{V}_{1 \mathrm{~b}}$, but not $\mathrm{V}_{2}$, vasopressin receptor genes are expressed in the supraoptic nucleus of the rat hypothalamus, and the transcripts are essentially colocalized in the vasopressinergic magnocellular neurons. Endocrinology 139:4701-4707.

Inenaga K, Yamashita H (1986) Excitation of neurones in the rat paraventricular nucleus in vitro by vasopressin and oxytocin. J Physiol (Lond) 370:165-180.

Ingram CD, Ciobanu R, Coculescu IL, Tanasescu R, Coculescu M, Mihai R (1998) Vasopressin neurotransmission and the control of circadian rhythms in the suprachiasmatic nucleus. Prog Brain Res 119:351-364.

Inouye SIT, Kawamura H (1979) Persistence of circadian rhythmicity in a mammalian hypothalamic "island" containing the suprachiasmatic nucleus. Proc Natl Acad Sci USA 76:5962-5966.

Jansen K, van der Zee EA, Gerkema MP (2000) Being circadian or not: vasopressin release in cultured SCN mirrors behavior in adult voles. NeuroReport 11:3555-3558.

Kalsbeek A, Buijs RM, van Heerikhuize JJ, Arts M, van der Woude TP (1992) Vasopressin-containing neurons of the suprachiasmatic nuclei inhibit corticosterone release. Brain Res 580:62-67.

Kalsbeek A, Buijs RM, Engelmann M, Wotjak CT, Landgraf R (1995) In vivo measurement of a diurnal variation in vasopressin release in the rat suprachiasmatic nucleus. Brain Res 682:75-82.

Kalsbeek A, Garidou ML, Palm IF, van der Vliet J, Simonneaux V, Pevet P, Buijs RM (2000) Melatonin sees the light: blocking GABA-ergic transmission in the paraventricular nucleus induces daytime secretion of melatonin. Eur J Neurosci 12:3146-3154.

Kruisbrink J, Mirmiram M, van der Woude TP, Boer GJ (1987) Effects of 
enhanced cerebrospinal fluid levels of vasopressin, vasopressin antagonist or vasoactive intestinal polypeptide on circadian sleep-wake rhythm in the rat. Brain Res 419:76-86.

Kubota A, Inouye SIT, Kawamura H (1981) Reversal of multiunit activity within and outside the suprachiasmatic nucleus in the rat. Neurosci Lett 27:303-308.

Lucas RJ, Hattar S, Takao M, Berson DM, Foster RG, Yau KW (2003) Diminished pupillary light reflex at high irradiances in melanopsinknockout mice. Science 299:245-247.

Meyer-Bernstein EL, Jetton AE, Matsumoto SI, Markuns JF, Lehman MN, Bittman EL (1999) Effects of suprachiasmatic transplants on circadian rhythms of neuroendocrine function in golden hamsters. Endocrinology 140:207-218.

Perreau-Lenz S, Kalsbeek A, Garidou ML, Wortel J, van der Vliet J, Van Heijningen C, Simonneaux V, Pevet P, Buijs RM (2003) Suprachiasmatic control of melatonin synthesis in rats: inhibitory and stimulatory mechanisms. Eur J Neurosci 17:221-228.

Ralph MR, Foster RG, Davis FC, Menaker M (1990) Transplanted suprachiasmatic nucleus determines circadian period. Science 247:975-978.
Reppert SM, Weaver DR (2001) Molecular analysis of mammalian circadian rhythms. Annu Rev Physiol 63:647-676.

Reppert SM, Weaver DR (2002) Coordination of circadian timing in mammals. Nature 418:935-941.

Schwartz WJ, Reppert SM (1985) Neural regulation of the circadian vasopressin rhythm in cerebrospinal fluid: a pre-eminent role for the suprachiasmatic nuclei. J Neurosci 5:2771-2778.

Shibata S, Moore RY (1993) Tetrodotoxin does not affect circadian rhythms in neuronal activity and metabolism in rodent suprachiasmatic nucleus in vitro. Brain Res 606:259-266.

Silver R, LeSauter J, Tresco PA, Lehman MN (1996) A diffusible coupling signal from the transplanted suprachiasmatic nucleus controlling circadian locomotor rhythms. Nature 382:810-813.

Vrang N, Larsen PJ, Moller M, Mikkelsen JD (1995) Topographical organization of the rat suprachiasmatic-paraventricular projection. J Comp Neurol 353:585-603.

Welsh DK, Logothetis DE, Meister M, Reppert SM (1995) Individual neurons dissociated from rat suprachiasmatic nucleus express independently phased circadian firing rhythms. Neuron 14:697-706. 